\section{Daten zum neuen CGRP-Antagonisten Ubrogepant bei akuter Migräne}

\author{
Ein neuer CGRP-Rezeptor-Antagonist ist in der klinischen Entwicklung. \\ Als Alternative zu Triptanen ist er etwa gleich effektiv, aber mit besserem \\ Verträglichkeitsprofil.
}

$S_{8}$ chon seit 20 Jahren befinden sich sogenannte CGRP-Rezeptor-Antagonisten als Alternative für die Triptane in der klinischen Entwicklung. Die ersten Substanzen wurden aus unterschiedlichen Gründen nicht weiterentwickelt, obwohl sie eine Wirksamkeit nachweisen konnten. Mit MK-1602 (Ubrogepant) liegt nun ein neuer CGRP-Rezeptor-Antagonist vor.

Die aktuelle doppelblinde und placebokontrollierte Dosisfindungsstudie randomisierte 834 Patienten, die je eine Migräneattacke behandelten. Die Dosis von Ubrogepant betrug $1 \mathrm{mg}, 10 \mathrm{mg}, 25$ $\mathrm{mg}, 50 \mathrm{mg}$ oder $100 \mathrm{mg}$. Die beiden primären Endpunkte waren Schmerzfrei- heit nach zwei Stunden und eine Besserung der Kopfschmerzen von schwer oder mittelschwer auf leicht oder keine Kopfschmerzen nach zwei Stunden. Insgesamt erhielten 527 Studienteilnehmer Ubrogepant und 113 Placebo.

Bezogen auf den Endpunkt Schmerzfreiheit nach zwei Stunden war der Unterschied ab der Dosis von 25 mg Ubrogepant zu Placebo statistisch signifikant. Unter 100 mg Ubrogepant waren 25,5\% der Patienten schmerzfrei im Vergleich zu 8,9\% unter Placebo. Die Besserung der Kopfschmerzen nach zwei Stunden war dagegen nicht signifikant unterschiedlich zu Placebo. In Bezug auf Nebenwirkungen bestanden keine signifi- kanten Unterschiede zwischen Ubrogepant und Placebo. Eine Erhöhung der Leberenzyme trat nicht auf.

\section{Kommentar}

Diese Studie zeigt eine dosisabhängige Wirkung des neuen CGRP-Rezeptor-Antagonisten Ubrogepant in der Therapie akuter Migräneattacken. Dabei liegt die Effektivität für die Schmerzfreiheit nach zwei Stunden etwa in der Größenordnung wie der der oralen Triptane. Das Verträglichkeitsprofil war allerdings formal gesehen besser als bei Triptanen, außerdem können CGRP-Rezeptor-Antagonisten auch bei kardiovaskulärem Risikoprofil eingesetzt werden. Die von der Firma MSD entwickelte Substanz wurde jüngst an die Firma Allergan verkauft, die nun in einem Phase 3-Studienprogramm die Substanz weiterentwickelt.

Prof. Dr. Dr. Stefan Evers

Voss T et al. A phase Ilb randomized, doubleblind, placebo-controlled trial of ubrogepant for the acute treatment of migraine. Cephalalgia 2016; 36: $887-98$

\title{
Botulinumtoxin bei Trigeminusneuralgie
}

\section{Patienten mit Trigeminusneuralgie, die nicht auf Carbamazepin und andere antikonvulsive Medikamente ansprechen, könnten laut einer Metaanalyse kleiner Studien eventuell von Botulinumtoxin profitieren.}

D ie klassische Trigeminusneuralgie ist zwar durch eine Reihe von Medikamenten wie etwa Carbamazepin gut zu beeinflussen, doch gibt es immer wieder Patienten, die auf diese Medikamente nicht ansprechen oder mit nicht tolerierbaren Nebenwirkungen reagieren. Es hat daher eine Reihe von Studien gegeben, einige auch placebokontrolliert, die die Wirksamkeit von Botulinumtoxin überprüft haben. Die Metaanalyse von Morra et al. fasst nun die Daten dieser placebokontrollierten Studien zusammen. Dabei konnten vier Studien mit insgesamt 178 Patienten identifiziert werden. Der Anteil der Responder lag bei Botulinumtoxin um den Faktor 2,9 (95\%-KI 1,8/4,7) höher als bei Placebo. Die mittlere Reduzierung der lanzinierenden Attacken war nur unter Botulinumtoxin mit $-29,8$ (95\%-KI -38,2/
-21,1) signifikant. Die Autoren folgern daraus, dass es deutliche Hinweise für eine Wirksamkeit von Botulinumtoxin bei Trigeminusneuralgie gibt.

\section{Kommentar}

Trotz der modernen antikonvulsiven Medikamente als Alternative zu Carbamazepin ist es weiterhin wichtig, weitere Therapieoptionen zur Behandlung der klassischen Trigeminusneuralgie zu entwickeln, da es immer noch zu viele Non-Responder gibt. Botulinumtoxin als wirksame Substanz ist dabei durchaus vorstellbar, da es dadurch zu einer Desensitisierung kommt, die schon in der Behandlung der chronischen Migräne ausgenutzt wird. Leider ist die Evidenz durch diese Metaanalyse eher schwach, da die einzelnen Studien klein waren und nur einen kurzen Beobachtungszeitraum umfassten. Angekündigt ist eine große rando- misierte Studie, die allerdings noch nicht vorliegt. Möglicherweise ist dann eine genauere Aussage möglich.

Prof. Dr. Dr. Stefan Evers

Morra ME, et al. Therapeutic efficacy and safety of Botulinum Toxin A Therapy in Trigeminal Neuralgia: a systematic review and meta-analysis of randomized controlled trials. J Headache Pain 2016; 17: 63

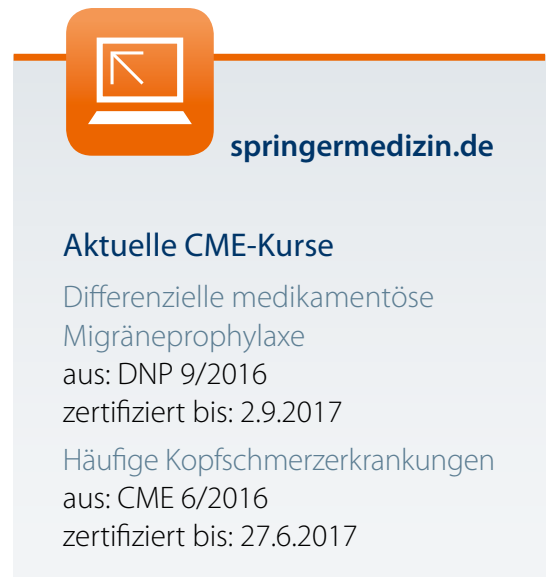

